\title{
New Results for Compton Scattering on Deuterium: A Better Determination of the Neutron Electromagnetic Polarizabilities
}

\author{
G. Feldman $^{\text {1a }}$, L.S. Myers ${ }^{b}$, J.R.M. Annand ${ }^{c}$, J. Brudvik ${ }^{d}$, K.G. Fissum ${ }^{d}$, \\ H.W. Griesshammer ${ }^{a}$, K. Hansen ${ }^{d}$, S.S. Henshaw ${ }^{\text {, }}$ L. Isaksson ${ }^{d}$, M.A. Kovash ${ }^{f}$, \\ M. Lundin ${ }^{d}$, J.A. McGovern ${ }^{g}$, A.M. Nathan ${ }^{b}$, D.R. Phillips ${ }^{h}$, B. Schröder ${ }^{d}$, \\ K. Shoniyozov ${ }^{f}$ and S.C. Stave ${ }^{e}$ \\ a George Washington University, Washington, DC, USA \\ b University of Illinois, Urbana, IL, USA \\ ${ }^{c}$ University of Glasgow, Glasgow, Scotland, UK \\ d Lund University, Lund, Sweden \\ e Duke University and TUNL, Durham, NC, USA \\ f University of Kentucky, Lexington, KY, USA \\ ${ }^{g}$ University of Manchester, Manchester, UK \\ h Ohio University, Athens, OH, USA
}

E-mail: feldman@gwu.edu, lmyers@jlab.org, kevin.fissum@nuclear.lu.se, hgrie@gwu.edu, lennart.isaksson@maxlab.lu.se, kovash@pa.uky.edu, judith.mcgovern@manchester.ac.uk, phillid1@ohio.edu

\begin{abstract}
The electric and magnetic polarizabilities of the proton are now well known, owing to Compton scattering measurements on hydrogen targets; however, the neutron polarizabilities are still relatively uncertain, compared to the proton values. To address this problem, a multiinstitutional collaboration has conducted a program of elastic Compton scattering experiments on deuterium at the MAX IV Laboratory in Lund, Sweden using tagged photons in the energy range $E_{\gamma}=65-115 \mathrm{MeV}$. We assembled at one laboratory, for the first time, three of the world's largest $\mathrm{NaI}$ detectors, each having better than $\Delta E / E \sim 2 \%$ photon energy resolution. We have measured elastic Compton scattering cross sections at lab angles of $\theta_{\gamma}=60^{\circ}, 120^{\circ}$ and $150^{\circ}$ over this energy range in $8 \mathrm{MeV}$ steps. This effectively doubles the world's set of elastic Compton scattering data from deuterium and provides valuable input for chiral effective field theory $(\chi$ EFT) calculations. These new deuterium data overlap previous measurements and extend them by $20 \mathrm{MeV}$ to higher energies. Based on $\chi$ EFT fits to the expanded world data set, new values for the neutron electric and magnetic polarizabilities have been obtained with greater accuracy than previously achieved, decreasing the statistical error by more than $30 \%$.
\end{abstract}

The 8th International Workshop on Chiral Dynamics, CD 2015

29 June 2015 - 03 July 2015

Pisa, Italy

\footnotetext{
${ }^{1}$ Speaker

(C) Copyright owned by the author(s) under the terms of the Creative Commons

Attribution-NonCommercial-NoDerivatives 4.0 International License (CC BY-NC-ND 4.0).
} 


\section{Introduction}

The electromagnetic polarizabilities of the nucleon (proton or neutron) are fundamental structure constants that characterize the extent to which a dipole moment (electric or magnetic) can be induced in that composite object as a result of an applied external field. These quantities are still not known satisfactorily, even to this day, although the values for the proton are in much better shape. Most of the work on this topic has been performed via Compton scattering experiments on the proton, exploiting the fact that there is an interference between the Thomson scattering term and the polarizability term in the scattering amplitude, and this leads to an enhancement of the polarizability contribution appearing in the expansion of the cross section: $\sigma_{p}(\omega) \approx r_{0}^{2}-2 r_{0} \alpha_{p} \omega^{2}$. A comprehensive discussion of the proton measurements can be found in the review paper by Griesshammer et al. [1], and the most recent extracted values for the proton polarizabilities have been presented in McGovern et al. [2].

For the neutron, the situation is considerably more limited, for several reasons. First of all, since the neutron is uncharged, there is no Thomson term in the scattering amplitude, and so for Compton scattering on a free neutron, the polarizability appears in the next order of the cross section expansion: $\sigma_{n}(\omega) \approx \alpha_{n}^{2} \omega^{4}$. Second of all, practically speaking, there are no free neutron targets, so the next best option is to use elastic Compton scattering on deuterium. This has the advantage of recovering the Thomson term (since the deuteron is charged), but it also has the disadvantage of providing only the sum of the proton and neutron polarizabilities: $\sigma_{D}(\omega) \approx r_{0}^{2}-2 r_{0}\left(\alpha_{p}+\alpha_{n}\right) \omega^{2}$. Nevertheless, this is under control in the treatment of the Compton scattering process in Chiral Effective Field Theory, and so the neutron experiments have primarily been carried out in this manner.

There have been other methods of investigating the neutron polarizabilities, namely lowenergy neutron scattering by a heavy nucleus and quasi-free Compton scattering on deuterium. These methods will not be discussed here, but these other experiments have also been summarized in the review paper in Ref. [1].

\section{Previous Experiments}

For the neutron case, there have been three principal elastic Compton scattering experiments: Lucas et al. (Illinois, 1994) at $E_{\gamma}=49$ and $69 \mathrm{MeV}$ [3], Hornidge et al. (Saskatoon, 2000) at $E_{\gamma}=95 \mathrm{MeV}$ [4], and Lundin et al. (Lund, 2003) at $E_{\gamma}=55$ and $66 \mathrm{MeV}$ [5]. These data are plotted in Fig. 1, and it is clear that the database is rather sparse. In comparison with the proton database, there are 29 neutron data points while there are 170 data points for the proton $[1,2]$. The neutron data cover a much narrower energy range $\left(E_{\gamma}=49-95\right.$ $\mathrm{MeV})$ compared to the proton data $\left(E_{\gamma}=40-170 \mathrm{MeV}\right)$, and the angular coverage is not so extensive. In fact, the lower-energy data of Lucas et al. and Lundin et al. essentially overlap in the forward-angle and backward-angle regions, and they cover almost the same photon energy bins. The higher-energy data set of Hornidge et al. is limited to only five data points. 


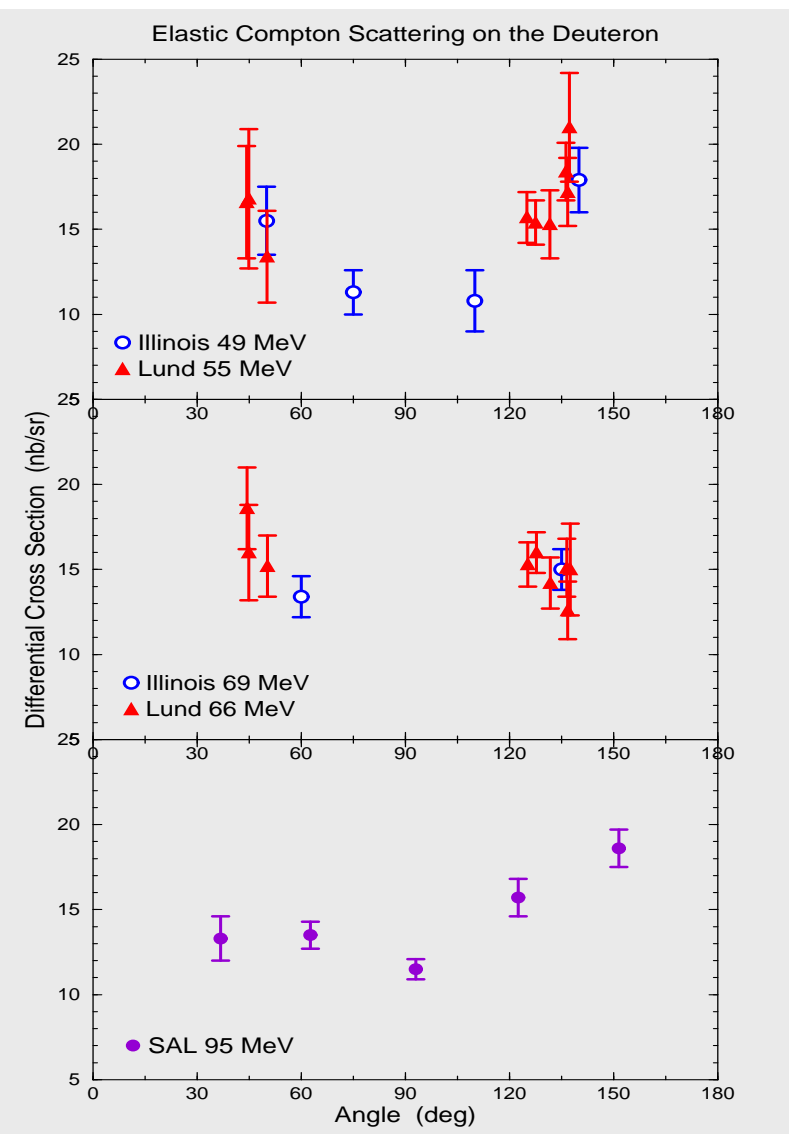

Figure 1: Angular distributions for the differential cross section of the $D(\gamma, \gamma) D$ elastic Compton scattering reaction in three different energy bins. The data are taken from Refs. [3-5].

\section{New Experiment at Lund}

We have addressed this problem by extending the deuteron Compton scattering experiments to higher energies (up to $115 \mathrm{MeV}$ ) at the MAX IV Laboratory in Lund, Sweden. We used three large $\mathrm{NaI}$ detectors (with $\sim 2 \%$ energy resolution) at angles of $60^{\circ}, 120^{\circ}$ and $150^{\circ}$ over the energy range $E_{\gamma}=65-115 \mathrm{MeV}$, which also included some overlap in energy with the previous measurements. In general, the kinematic coverage of our measurement, along with that of the earlier Compton scattering experiments, is shown in Fig. 2.

The experimental setup is shown in Fig. 3. An electron beam from the MAX I pulsestretcher ring impinges on a metallic radiator to produce bremsstrahlung photons. The converted electrons are momentum-analyzed in the tagging spectrometer [6] and then detected in an array of thin plastic scintillators in the focal plane. The unconverted electron beam is directed into a distant beam dump. The bremsstrahlung photons continue in a straight trajectory downstream, through a defining collimator, and then impinge on the liquid deuterium $\left(\mathrm{LD}_{2}\right)$ target located on the other side of thick concrete shielding walls (protecting the target area from 
the photon tagger location). The three NaI detectors (BUNI, CATS and DIANA) surround the $\mathrm{LD}_{2}$ target, and a coincidence between a detected photon in one of the NaI's and an electron hit in the tagger focal-plane array is what defines a "tagged photon" event in the data stream.

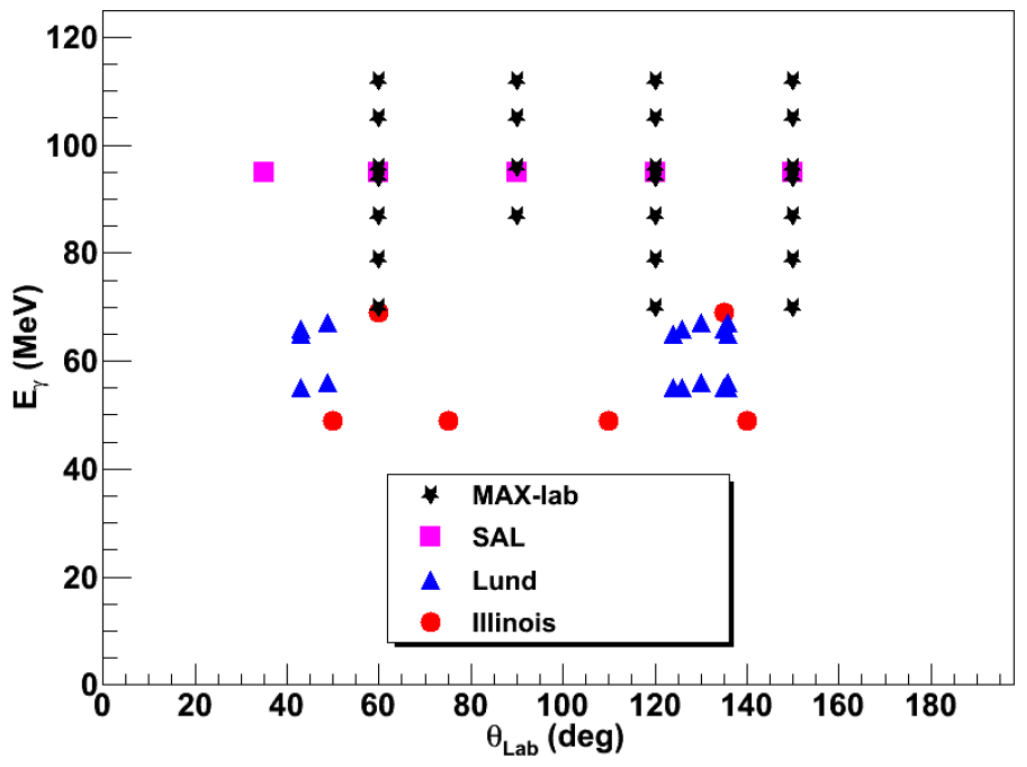

Figure 2: Kinematic coverage (energy and angle) for the current experiment and the three previous experiments [3-5] for elastic Compton scattering on deuterium.

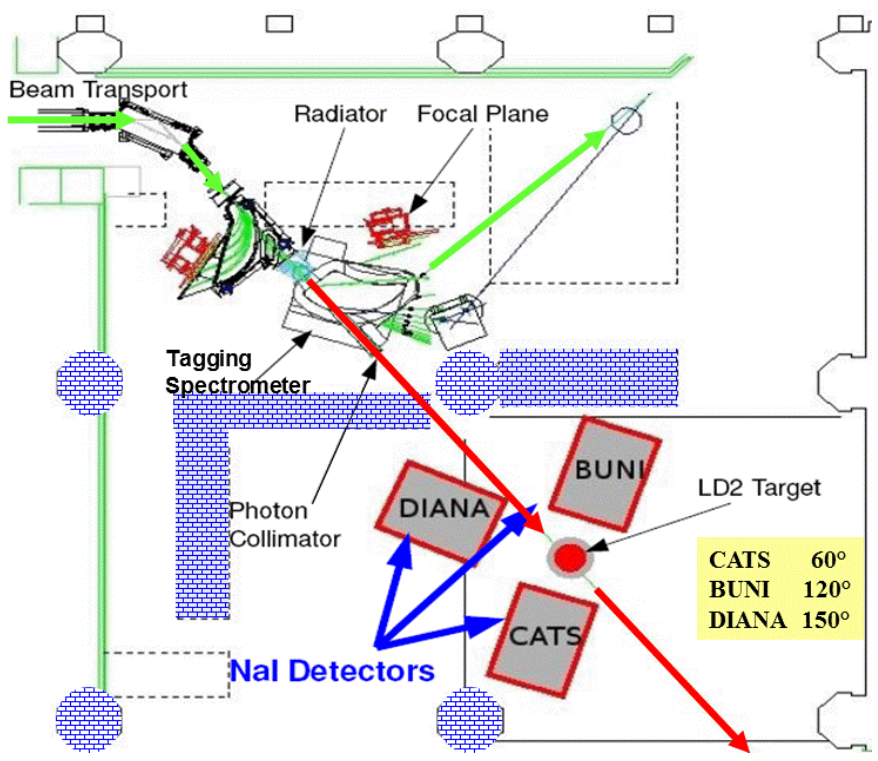

Figure 3: Experimental setup for the photon tagger and the detector configuration for the deuterium Compton scattering experiment at the MAX IV Laboratory. 
The timing information is shown in the TDC spectrum depicted in Fig. 4a. The true coincidence peak (prompt peak) highlighted in red is clearly visible, although it is surrounded by a highly structured background that was actually very difficult to characterize. After much work, a Monte Carlo simulation of the tagger electronics and the beam time structure was developed [7] to tackle this problem, and finally we were successful in determining the shape of the random background underneath the true coincidence (prompt) peak, as shown in Fig. 4b.
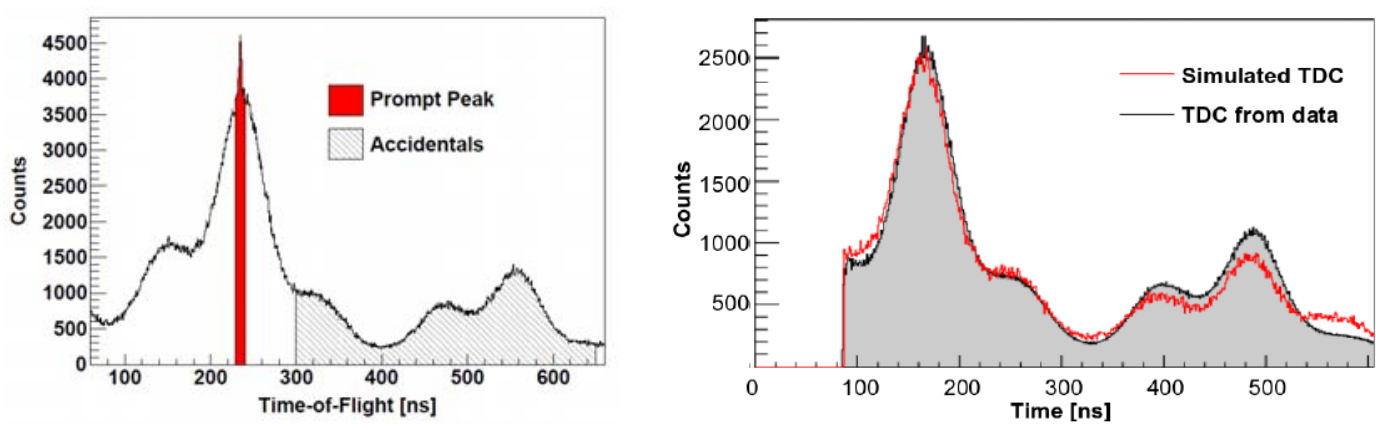

Figure 4: Left panel (a): Time spectrum showing the tagger coincidence (prompt) peak and the random background. Right panel (b): Comparison of the Monte Carlo simulation of the tagger with a real experimental time spectrum. Details of the simulation can be found in Ref. [7].

Using the timing information to generate a prompt energy spectrum and the corresponding background energy spectrum for random coincidences, we were able to determine the experimental yield by subtracting the background, fitting a NaI lineshape to the elastic scattering peak, and summing the data over a fixed energy integration region $( \pm 2 \mathrm{MeV}$ around the elastic peak). The plot in Fig. 5a shows the yield as a function of missing energy, where the latter is determined by the difference between the detected photon energy (measured in the NaI) and the expected photon energy (based on kinematics, using the incident photon energy and scattering angle). The elastic peak should appear at a missing-energy value of zero, as seen in the plot. The lineshape fit from our NaI detector GEANT simulation is also shown.

As a cross-check of our normalization for the absolute cross section, we typically run for about 24 hours on a solid carbon target, and we compare our extracted carbon cross section to the results in the literature. To illustrate the difficulty of the deuterium Compton scattering experiment, it is interesting to examine the spectra from carbon and deuterium together, as shown in Fig. 5b. The carbon yields almost as many counts in one day as the deuterium does in 15 days. Also, as a rough comparison, we see that the carbon signal/noise ratio at the peak is about $2800 / 1400=2 / 1$, whereas the deuterium signal/noise ratio is $2800 / 2200=1.3 / 1$. 

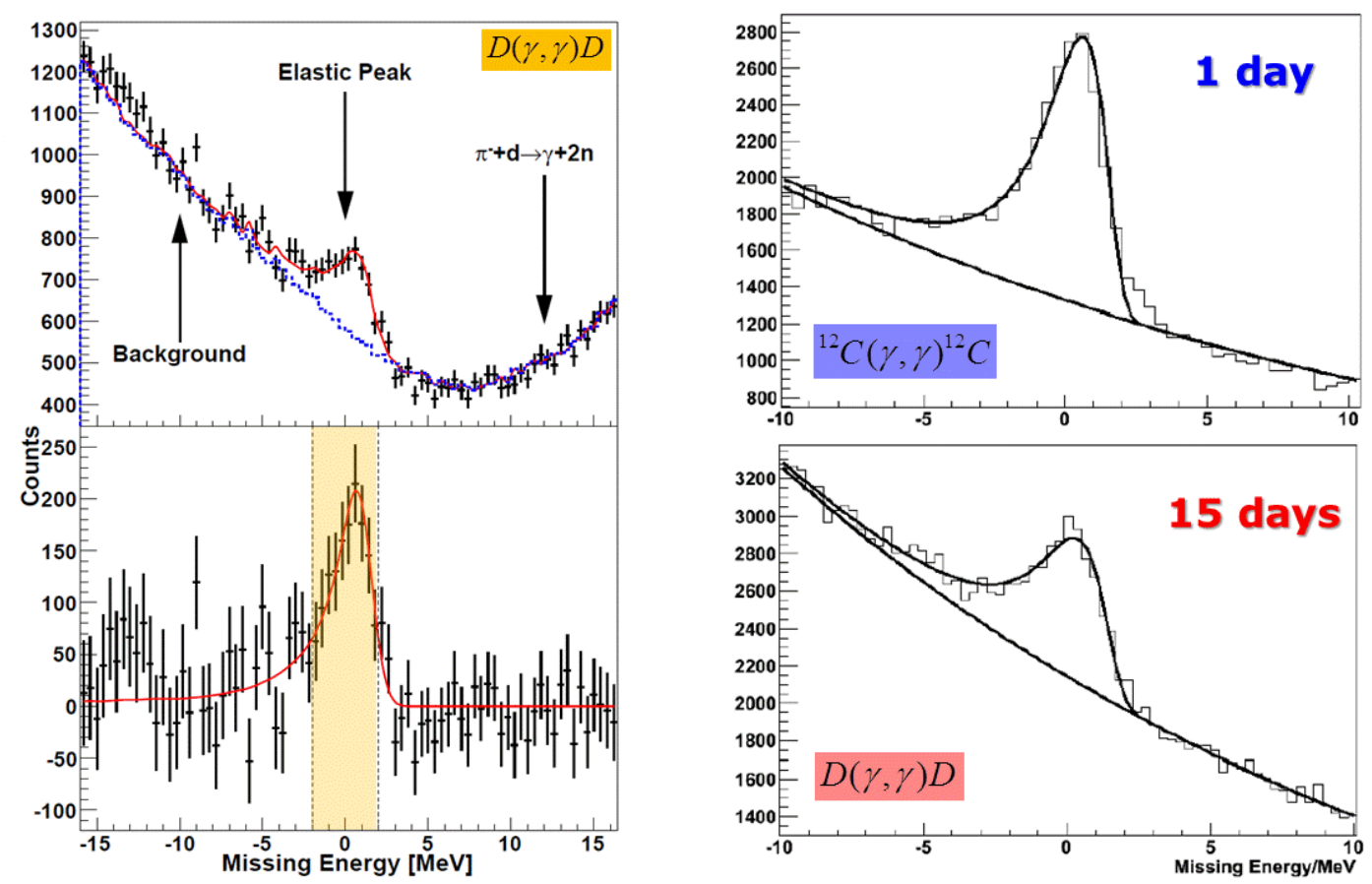

Figure 5: Left panel (a): Missing-energy spectrum for the $D(\gamma, \gamma) D$ reaction, showing the elastic scattering peak and the background due to random coincidences. The bottom panel shows the background-subtracted spectrum, with the summing region and the lineshape fit to the data. Right panel (b): Comparison of missing-energy spectra for Compton scattering from carbon (top) and deuterium (bottom).

The results for our carbon cross-check are shown in Fig. 6, where the differential cross section is plotted as a function of photon energy. Over the energy range of the new carbon data, we have good agreement with the previous results at the three angles of our measurement. More details about the carbon data can be found in Ref. [8]. This enables us to assert that we are confident in our normalization procedure, and we can now take a look at the deuterium Compton scattering cross section which is the primary objective of our experiment.

Our final deuterium results are presented in Fig. 7, where the differential cross section is plotted as a function of photon energy. Also, shown in the figure are the previous data from Lucas [3] and Lundin [5] (only the higher-energy data points from those experiments are included) and the Hornidge data at $95 \mathrm{MeV}$ [4]. Our results are in good agreement with these earlier results, and clearly the new data fill in a great deal of the kinematic range. The curves are from Chiral Effective Field Theory $(\chi E F T)$ calculations by Griesshammer which are described in another paper in this same session (also see Refs. [9-10] for more details). It is from these $\chi$ EFT fits to the data that the neutron polarizabilities were extracted. In Fig. 8, we show the specific angular distributions for various energy bins starting at the lowest photon energy of $49 \mathrm{MeV}$ - the Lucas and Lundin data only occupy the first three frames, the Hornidge data only appear in the $95 \mathrm{MeV}$ frame, and the Myers data start at $66 \mathrm{MeV}$. 


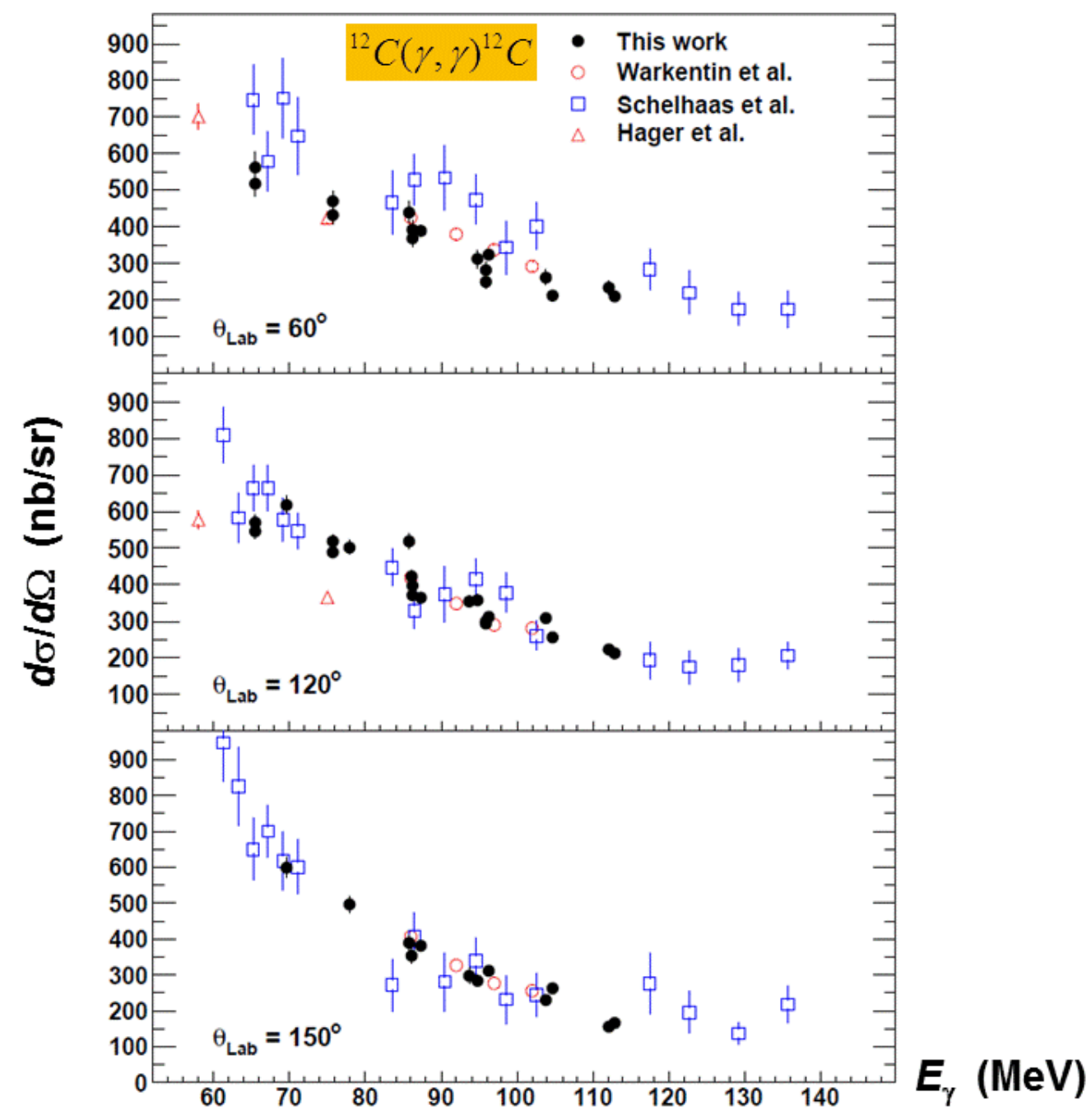

Figure 6: Differential cross sections as a function of energy for the ${ }^{12} C(\gamma, \gamma)^{12} C$ reaction at three different laboratory angles. The current data are compared to previous measurements in the literature in order to provide a cross-check of our absolute normalization. More details of these new carbon measurements can be found in Ref. [8]. 


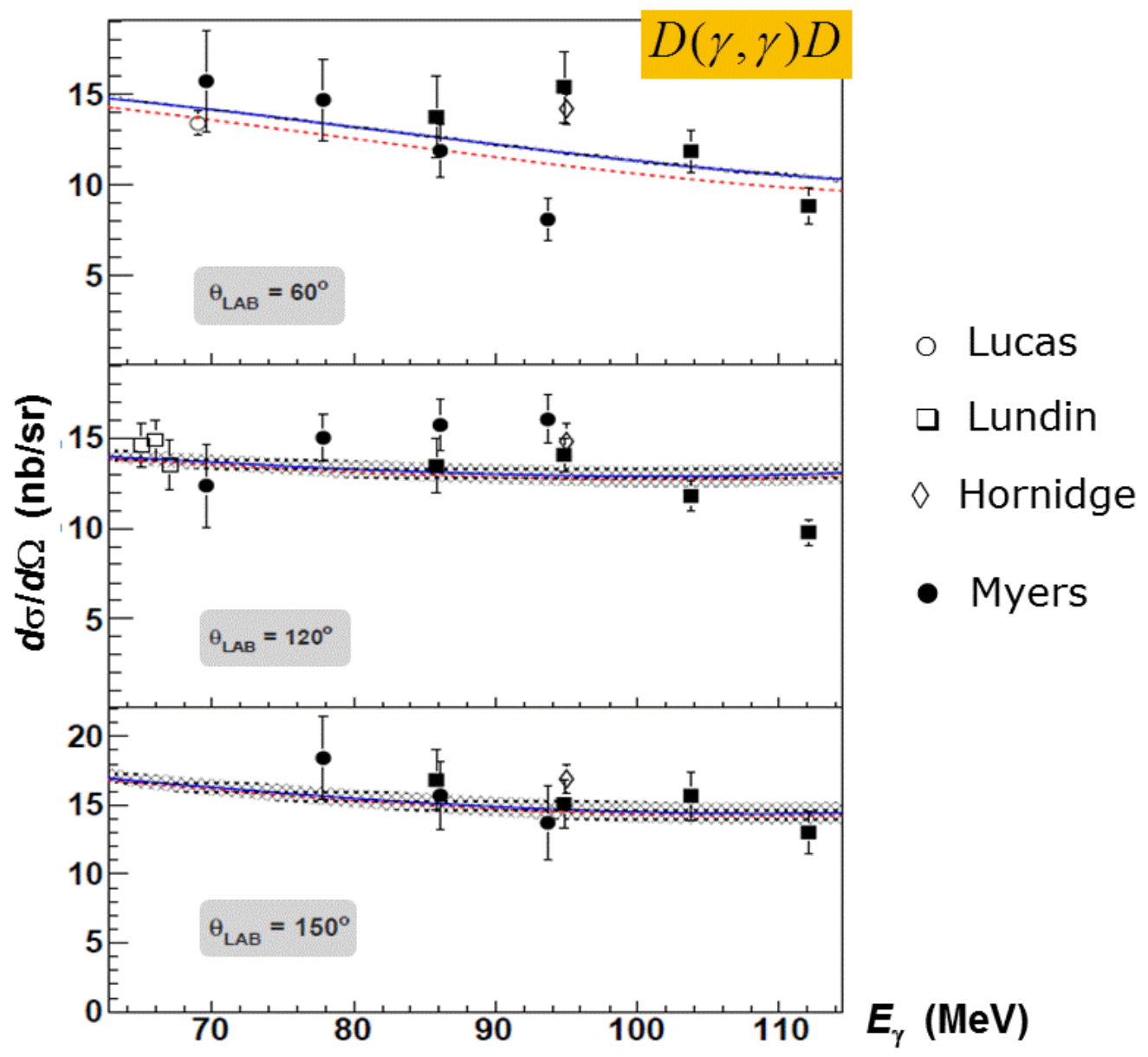

Figure 7: Differential cross sections as a function of energy for the $D(\gamma, \gamma) D$ reaction at three different laboratory angles. The results from earlier measurements [3-5] are also shown. The curves are $\chi$ EFT fits to the global data set, taken from Ref. [10]. 


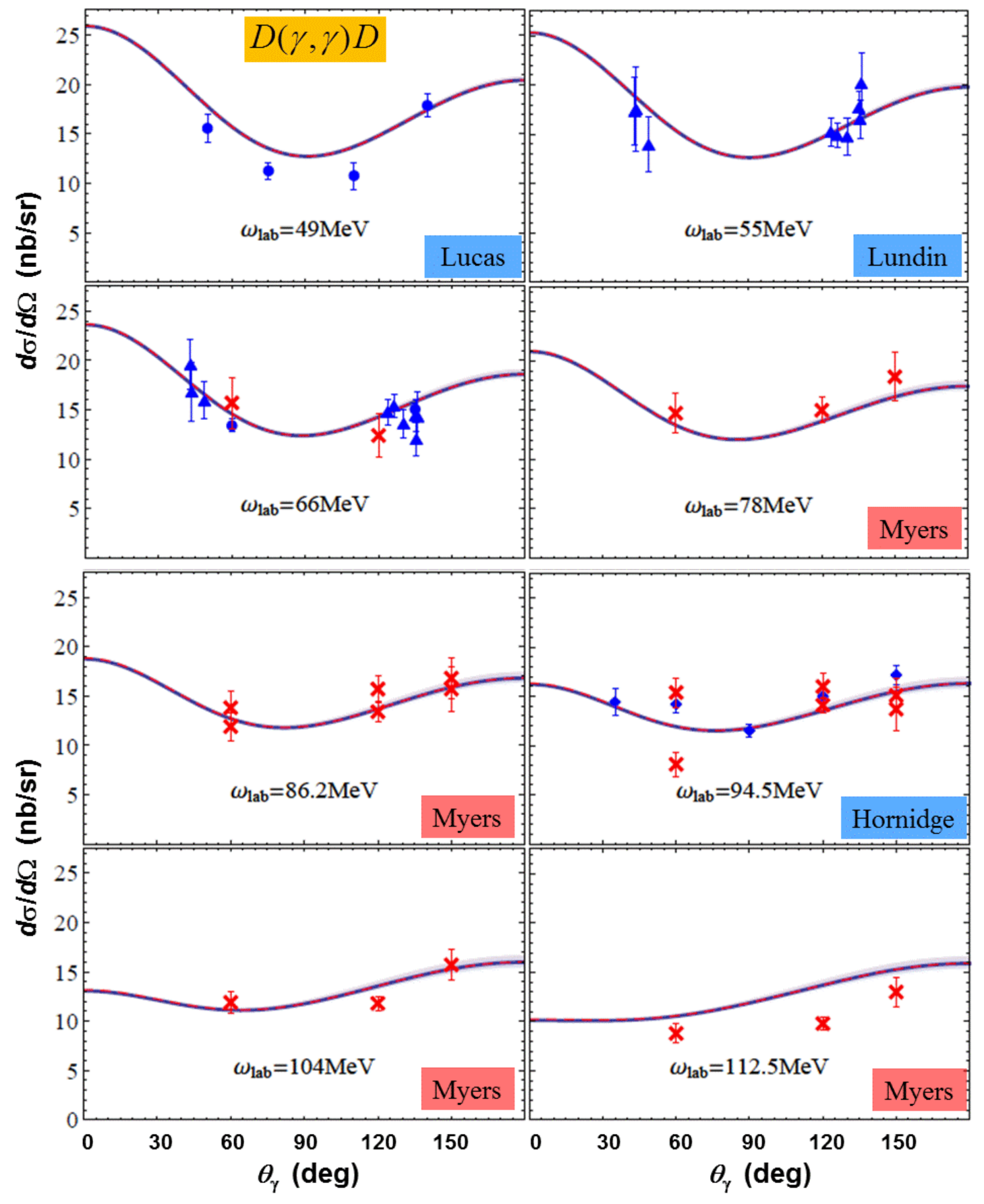

Figure 8: Angular distributions for the differential cross section of the $D(\gamma, \gamma) D$ reaction in specific energy bins spanning the entire range of the current and previous measurements. The curves are $\chi$ EFT fits to the global data set, taken from Ref. [9]. 


\section{Summary}

The current data set has roughly doubled the number of deuterium elastic Compton scattering points in the world database and has extended the world data set to higher energy and more backward angles. From the $\chi E F T$ fits to the global data set (including Lucas, Lundin, Hornidge and Myers data), the latest results for the neutron polarizabilities are [9]:

$$
\begin{gathered}
\alpha_{n}=11.55 \pm 1.25(\text { stat }) \pm 0.2(B S R) \pm 0.8(\text { theory }) \\
\beta_{n}=3.65 \mp 1.25(\text { stat }) \mp 0.2(B S R) \mp 0.8(\text { theory })
\end{gathered}
$$

which constitutes a $30 \%$ reduction in the statistical uncertainty compared to the previously published values of the polarizabilities. The uncertainties for $\alpha_{n}$ and $\beta_{n}$ are anti-correlated due to imposing the Baldin Sum Rule (BSR) condition for $\alpha_{n}+\beta_{n}$ as a constraint in the $\chi$ EFT fitting procedure. More details about the data acquisition and analysis, as well as the theoretical calculations, can be found in Refs. [9-10].

There are still more data from Lund yet to come - these new data focus exclusively on the higher-energy region $\left(E_{\gamma}=81-115 \mathrm{MeV}\right)$ where the Compton scattering cross section is more sensitive to the polarizabilities. The new data set also includes $90^{\circ}$ and should have smaller rate-dependent corrections due to improved beam quality at the MAX IV facility. These data are currently under analysis and will potentially contribute as many new data points as the Myers data set that was reported in the current paper.

\section{References}

[1] H.W. Griesshammer, J.A. McGovern, D.R. Phillips and G. Feldman, Prog. Part. Nucl. Phys. 67, 841 (2012).

[2] J.A. McGovern, D.R. Phillips and H.W. Griesshammer, Eur. Phys. J. A49, 12 (2013).

[3] M.A. Lucas, Ph.D. dissertation, University of Illinois (1994).

[4] D.L. Hornidge, B.J. Warkentin, R. Igarashi, J.C. Bergstrom, E.L. Hallin, N.R. Kolb, R.E. Pywell, D.M. Skopik, J.M. Vogt and G. Feldman, Phys. Rev. Lett. 84, 2334 (2000).

[5] M. Lundin et al., Phys. Rev. Lett. 90, 192501 (2003).

[6] J.O. Adler et al., Nucl. Instrum. Meth. Phys. Res. A715, 1 (2013).

[7] L.S. Myers, G. Feldman, K.G. Fissum, L. Isaksson, M.A. Kovash, A.M. Nathan, R.E. Pywell and B. Schröder, Nucl. Instrum. Meth. Phys. Res. A729, 707 (2013).

[8] L.S. Myers et al., Phys. Rev. C89, 035202 (2014).

[9] L.S. Myers et al., Phys. Rev. Lett. 113, 262506 (2014).

[10] L.S. Myers et al., Phys. Rev. C92, 025203 (2015). 